\title{
Secondary Metabolite Profile and Maize Weevil Repellent Activity of the Leaves of Ruta graveolens
}

\author{
Perera A.G.W.U. ${ }^{1 *}$, Karunaratne M.M.S.C. ${ }^{1}$ and Chinthaka S.D.M. ${ }^{2}$ \\ ${ }^{I}$ Department of Zoology, University of Sri Jayewardenepura, Sri Lanka \\ ${ }^{2}$ Department of Chemistry, University of Sri Jayewardenepura, Sri Lanka \\ *wathsalauda@gmail.com
}

\begin{abstract}
Secondary metabolites which make the major contribution to specific odors and flavors of plants play a critical role when dealing as repellents against insects in storage pest management. Thus, identification of those secondary metabolites that have repulsive effect on insect pest behaviors could greatly facilitate the development of green pesticides while ensuring ecological sustainability. Hence, the present phytocentric research study provides the first investigation on volatile profile, chemical composition and repellent potential of the leaves of $R$. graveolens (Aruda) against the maize weevil, Sitophilus zeamais (Motschulsky) infestations. The volatile organic compound (VOC) profile of fresh leaves of $R$. graveolens was analyzed with headspace solid-phase microextraction coupled with gas chromatography and mass spectrometry (HSSPME/GC-MS). Qualitative phytochemical screening of 11 phytoconstituents (alkaloids, saponins, flavonoids, tannins, steroids, terpenoids, anthraquinones, glycosides, phlobatannins, coumarins and phenols) for four leaf extracts (aqueous, n-hexane, ethyl acetate and methanol) was also performed following the standard procedures. In addition, contact and fumigant repellent properties of $R$. graveolens were also evaluated. A total of 14 compounds representing $99.95 \%$ of the total quantum of volatiles were detected in $R$. graveolens leaves consisting mainly of 2-ketones (93.32\%), sesquiterpenoids (1.48\%), aliphatic acids and esters (1.29\%): 2Nonanone $(25.71 \%)$ and 2-Undecanone $(55.51 \%)$ were the major volatile compounds followed by 2-Decanone $(5.60 \%), 5$, 6-diethenyl-1-methyl-cyclohexene (3.76\%), 2-Dodecanone $(2.74 \%)$ and 2-Tridecanone (2.98\%). Phytochemical results revealed the absence of phlobatannins and the presence of alkaloids, glycosides and coumarins in each leaf extract. Aqueous leaf extract exhibited the presence for majority of phytoconstituents while hexane demonstrating the least presence. The data on contact and fumigation repellency against $S$. zeamais indicated strong maize grain protection activities at the highest dosage accounting for $97 \%$ and $96 \%$ respectively. The present study provides the evidence that $R$. graveolens leaves contain agriculturally important bioactive secondary plant metabolites, thus justifying the use of plant species with respect to its significant repellent role against $S$. zeamais populations in post-harvest storage pest management programs.
\end{abstract}

Keywords: Ruta graveolens, Sitophilus zeamais, Secondary metabolites, Repellency, Headspace-solid-phase micro-extraction

Proceedings of the International Forestry and Environment Symposium 2016, Department of Forestry and Environmental Science, University of Sri Jayewardenepura, Sri Lanka. 